\title{
Static Behavior Prediction of Microelectrostatic Actuators by Discrete Geometric Approaches
}

\author{
P. Bettini, E. Brusa, M. Munteanu, R. Specogna, and F. Trevisan \\ Dipartimento di Ingegneria Elettrica, Gestionale e Meccanica, Università of Udine, I-33100 Udine, Italy
}

The analysis of a microelectrostatic actuator is presented, formulated by means of the so-called Discrete Geometric Approach applied to the solution of the electrostatic-elastostatic coupled problem. Numerical results computed by means of the proposed approach are compared to those coming from assessed approaches like the finite-element method (FEM) (for both the structural and the electric domains) and FEM/boundary element method (BEM) (FEM for the structure, BEM for the electric domain). A preliminary experimental validation is finally added.

Index Terms-Discrete geometric approach, finite elements, microelectromechanical systems (MEMS).

\section{INTRODUCTION}

$\mathbf{O}$ NE OF the most challenging advances in microsystem design is an efficient use of electromechanical coupling for actuation, sensing, and energy harvesting purposes. Scale laws demonstrate that electric field applied to flexible microstructures provides stronger actuation, if compared to magnetic forces [1]. Therefore microbeam electrostatic actuators are widely used in radio-frequency microelectromechanical systems (MEMS), although they suffer breakdown voltage and pull-in effect, both causing the structural collapse. Electrostatic force exhibits a nonlinearity on voltage, charge, and mechanical displacement, which makes difficult a straight and fast computation of stress, strain, and displacement to foresee the mechanical reliability [2].

The aim of this work is to investigate the capability of the so-called Discrete Geometric Approach (DGA) [3]-[6] in the solution of a plain, static, and nonlinear electrostatic-elastostatic coupled problem to compute displacements and voltage at pull-in on a set of cantilever microbeams. In this way, we use a unique approach for both the electrical and the mechanical domains, with a benefit for the design of an efficient numerical tool; in addition, we propose second-order triangular elements for both the domains.

A sequential approach has been adopted [7], by solving the electric and mechanical problems separately. The numerical results are compared to the experimental ones obtained on a set of cantilever microbeams with several geometrical aspect ratios, including length, width, gap, and thickness.

The computational efficiency of this method is compared to the nonlinear solutions of the sequential approach when the electrical and mechanical problems have been discretized both by the finite-element method (FEM) or even by FEM in the structure and boundary element method (BEM) in the dielectric; in addition, a special mechanical finite element [8] has been also considered.

Digital Object Identifier 10.1109/TMAG.2007.916393

Color versions of one or more of the figures in this paper are available online at http://ieeexplore.ieee.org.

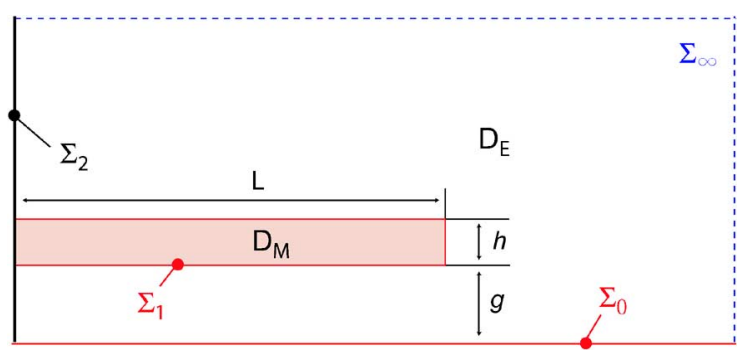

Fig. 1. Sketch of the 2-D electrical $\left(D_{E}\right)$ and mechanical $\left(D_{M}\right)$ domains (not on scale). The boundary $\Sigma_{1}$ and $\Sigma_{0}$ of the conducting domain is shown in addition. $\Sigma_{\infty}$ is the truncation of the infinity domain of the electrostatic problem. Finally, on $\Sigma_{2}$ the Neumann boundary condition is imposed.

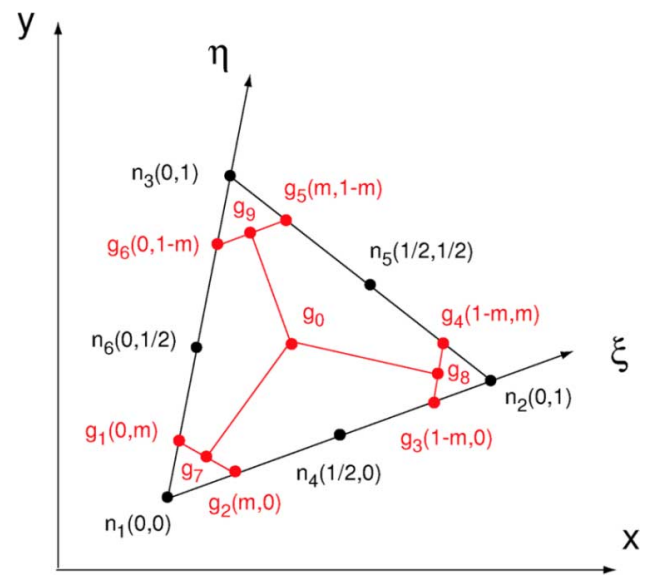

Fig. 2. Global Cartesian coordinate system $(x, y)$ and local affine coordinate system $(\xi, \eta)$ for the triangle $t$. Special Gauss points $g_{i}, i=1, \ldots, 6$ on the edges of the triangle are shown. Finally $g_{0}$ denotes the center of mass of $t$.

\section{Discrete APPROACH FOR THE COUPLED PROBLEM}

A sketch of the 2-D domain geometry is shown in Fig. 1 for the electromechanical coupled problem. The domain of interest have been partitioned into the mechanical $D_{M}$ and the electrical $D_{E}$ domains. In $D_{M} \cup D_{E}$, we will introduce a pair of interlocked cell complexes based on triangles, [3], [9]. Without loosing generality, we focus on a triangular element $t$, Fig. 2. The primal complex consists of the nodes $n_{1}, n_{2}, n_{3}$ of $t$, and of the additional nodes $n_{4}, n_{5}, n_{6}$ forming the center of mass of the edges of $t$. The primal edges are obtained by splitting in two halves each of the edges of $t$. In order to assure a second-order 
convergence in terms of electric field $\mathrm{E}$ and electric flux density $D$ in $D_{E}$, or in terms of strain $\varepsilon_{x}, \varepsilon_{y}, \gamma$ and stress $\sigma_{x}, \sigma_{y}, \tau$ components in $D_{M}$, we will build a dual-cell complex starting from special points in each of the edges of $t$ [5]; these points coincide with the pair of points for the entire edge of $t$ of a Gauss integration formula of second degree. We denote with $\left(x_{i}, y_{i}\right)$, with $i=1, \ldots, 6$, the Cartesian global coordinates of node $n_{i}$. We introduce in $t$ a local affine reference frame $(\xi, \eta)$ as shown in Fig. 2. The relation between the local coordinates $(\xi, \eta)$ of a point in $t$ and the corresponding global coordinates $(x, y)$ is

$$
\left[\begin{array}{l}
x \\
y
\end{array}\right]=\left[\begin{array}{l}
x_{1} \\
y_{1}
\end{array}\right]+\mathbf{T}\left[\begin{array}{l}
\xi \\
\eta
\end{array}\right]
$$

where the transformation matrix $\mathbf{T}$ is defined as

$$
\mathbf{T}=\left[\begin{array}{cc}
x_{2}-x_{1} & x_{3}-x_{1} \\
y_{2}-y_{1} & y_{3}-y_{1}
\end{array}\right]
$$

Along each of the edges of $t$ we denote with $g_{h}, g_{k}$ the pair of Gauss points. For example, with reference to the edge from $n_{1}$ to $n_{2}$, we write $g_{1}=(m, 0), g_{2}=(1-m, 0)$, with $m=$ $(1 / 2)-(1 / 2 \sqrt{3})$. The center of mass of $t$ is denoted with $g_{0}$. The center of mass of the edges between the pair of points $\left(g_{1}\right.$, $\left.g_{2}\right),\left(g_{3}, g_{4}\right),\left(g_{5}, g_{6}\right)$ are denoted with $g_{7}, g_{8}, g_{9}$, respectively.

In our 2-D model, a dual surface $\tilde{s}_{h k}$ has a unitary thickness in the out of plane direction and its trace is a line segment from node $g_{h}\left(\xi_{h}, \eta_{h}\right)$ to node $g_{k}\left(\xi_{k}, \eta_{k}\right)$. The components $\left(\tilde{s}_{x}, \tilde{s}_{y}\right)$ of the area vector ${ }^{1} \tilde{\mathrm{S}}_{h k}$ of $\tilde{s}_{h k}$ are defined as

$$
\left[\begin{array}{c}
\tilde{s}_{x} \\
\tilde{s}_{y}
\end{array}\right]=\left[\begin{array}{cc}
0 & -1 \\
1 & 0
\end{array}\right] \mathbf{T}\left[\begin{array}{l}
\xi_{k}-\xi_{h} \\
\eta_{k}-\eta_{h}
\end{array}\right] \text {. }
$$

Next, we introduce a dual volume $\tilde{v}_{i}$, in a one to one correspondence with $n_{i}$. Its boundary is a collection of $\tilde{s}_{h k}$ facing $n_{i}$ and it is oriented by the outer normal. For example (refer to Fig. 2), for $n_{4}, \tilde{v}_{4}$ is bounded by $\tilde{s}_{27}, \tilde{s}_{70}, \tilde{s}_{08}$, and $\tilde{s}_{83}$.

\section{A. Formulation of the DGA for Electrostatics}

For each $t \in D_{E}$, the DGA for electrostatics can be cast in terms of the electric potential $V$ (associated with a primal node $n$ ) together with the electric flux $\Psi$ (associated with a dual face $\tilde{s}$ ) and the electric charge $Q$ (associated with a dual volume $\tilde{v}$ ). We assume here that no free charge is present in $D_{E}$, and then $Q=0$ for each $\tilde{v}_{i}$.

In the local coordinate system $(\xi, \eta), V$ is approximated with a second-order polynomial

$$
V(\xi, \eta)=\left[\begin{array}{llllll}
1 & \xi & \eta & \xi^{2} & \xi \eta & \eta^{2}
\end{array}\right]\left[a_{1} \ldots a_{6}\right]^{T}
$$

where the coefficients $\mathbf{a}=\left[a_{1} \ldots a_{6}\right]^{T}$ can be computed in terms of the electric voltages $\mathbf{V}=\left[V_{1} \ldots V_{6}\right]^{T}$ at the six primal nodes of $t$; in this way we obtain

$$
\mathbf{a}=\mathbf{C V}
$$

\footnotetext{
${ }^{1}$ The area vector has amplitude equal to the area of $\tilde{s}_{h k}$ and it is normal to $\tilde{s}_{h k}$ with a specified orientation.
}

where $\mathbf{C}$ is the resulting $6 \times 6$ matrix. Then, $V$ can be expressed as

$$
V(\xi, \eta)=\left[\begin{array}{llllll}
1 & \xi & \eta & \xi^{2} & \xi \eta & \eta^{2}
\end{array}\right] \mathbf{C V} .
$$

From (6), the components of $\mathrm{E}$ along $(x, y)$ are

$$
\left[\begin{array}{l}
E_{x} \\
E_{y}
\end{array}\right]=-\left[\begin{array}{l}
\partial_{x} V \\
\partial_{y} V
\end{array}\right]=-\mathbf{J}\left[\begin{array}{l}
\partial_{\xi} V \\
\partial_{\eta} V
\end{array}\right]=-\left(\mathbf{T}^{-1}\right)^{T} \mathbf{N C V}
$$

where $\mathbf{J}$ is the Jacobian matrix of the mapping from $(\xi, \eta)$ to $(x, y)$ coordinates, and the matrix $\mathbf{N}$ is defined as

$$
\mathbf{N}=\left[\begin{array}{cccccc}
0 & 1 & 0 & 2 \xi & \eta & 0 \\
0 & 0 & 1 & 0 & \xi & 2 \eta
\end{array}\right]
$$

Denoting with $\Psi_{h k}=\int_{\tilde{s}_{h k}} D \cdot d s$ the electric flux of $D$ through $\tilde{s}_{h k}$, we get

$$
\Psi_{h k}=\left[\begin{array}{ll}
\tilde{s}_{x} & \tilde{s}_{y}
\end{array}\right]\left[\begin{array}{l}
D_{x} \\
D_{y}
\end{array}\right]
$$

where $D_{x}$ and $D_{y}$ are the components of $D$ evaluated in the center of mass of $\tilde{s}_{h k}$. By substituting in (9), (3) for the components of $\tilde{\mathrm{s}}_{h k}$, using the constitutive relation $D=\varepsilon \mathrm{E}$, with $\varepsilon$ the uniform permittivity of $t$, and evaluating the components of $\mathrm{E}$ according to (7), we obtain

$$
\Psi_{h k}=-\tilde{\mathbf{s}}_{h k} \varepsilon\left(\mathbf{T}^{-1}\right)^{T} \mathbf{N C V}
$$

where $\varepsilon$ is a constant for homogeneous, isotropic, and linear dielectric material inside each primal cell, or it is a tensor in the general case.

Next, for element $t$ we may write the local contribution to Gauss' Law as

$$
\tilde{\mathbf{D}} \Psi=0
$$

where $\tilde{\mathbf{D}}$ is the matrix of incidence numbers between the outer orientation of $\tilde{v}_{i}$ and the outer orientation of $\tilde{s}_{h k}$.

Finally, assembling the contribution from (11) for each $t$, the final system becomes

$$
\mathbf{K}_{E} \mathbf{V}=0
$$

where $\mathbf{K}_{E}$ is the resulting stiffness matrix for the electrostatic problem. The boundary conditions must be assigned to close the problem in $D_{E}$, by prescribing $V_{1}=V$ for the potential on $\Sigma_{1}$, $V_{0}=0$ for the potential on $\Sigma_{0} \cup \Sigma_{\infty}$, and zero electric flux on $\Sigma_{2}$.

From the solution of (12), the components of the electrostatic force acting on $n_{i}$ laying on $\Sigma_{1}$ can be evaluated as

$$
\left[\begin{array}{l}
F_{x} \\
F_{y}
\end{array}\right]=\frac{1}{2} Q_{i}\left[\begin{array}{c}
E_{a x} \\
E_{a y}
\end{array}\right]
$$

where $Q_{i}$ is the surface charge lying on the conducting boundary $\Sigma_{1}$ in the neighborhood of node $n_{i}$ and $E_{a x}, E_{a y}$ are the components of the average electrical field acting on $n_{i}$. 


\section{B. Formulation of the DGA for Elastostatics}

For each $t \in D_{M}$, the DGA for elastostatics can be cast in terms of the components $U_{x}$ and $U_{y}$ of the displacement vector (associated with a primal node $n$ ), together with the surface force vector $\mathrm{F}$ (associated with a dual face $\tilde{s}$ ). Again, we approximate $U_{x}, U_{y}$ as

$$
\left[\begin{array}{ll}
U_{x} & U_{y}
\end{array}\right]=\left[\begin{array}{llllll}
1 & \xi & \eta & \xi^{2} & \xi \eta & \eta^{2}
\end{array}\right] \mathbf{C}\left[\begin{array}{lll}
\mathbf{U}_{x} & \mathbf{U}_{y}
\end{array}\right]
$$

where $\mathbf{U}_{x}, \mathbf{U}_{y}$ are the arrays of the displacement components in the primal nodes. From (14), the displacement gradient components along the axes of the global coordinate system $(x, y)$ are

$$
\left[\begin{array}{cc}
\partial_{x} U_{x} & \partial_{x} U_{y} \\
\partial_{y} U_{x} & \partial_{y} U_{y}
\end{array}\right]=\left[\begin{array}{l}
\mathbf{B}_{1} \\
\mathbf{B}_{2}
\end{array}\right]\left[\begin{array}{ll}
\mathbf{U}_{x} & \mathbf{U}_{y}
\end{array}\right]
$$

where

$$
\left[\begin{array}{l}
\mathbf{B}_{1} \\
\mathbf{B}_{2}
\end{array}\right]=\left(\mathbf{T}^{-1}\right)^{T} \mathbf{N C}
$$

and the strain components can be written as

$$
\left[\begin{array}{c}
\varepsilon_{x} \\
\varepsilon_{y} \\
\gamma
\end{array}\right]=\left[\begin{array}{cc}
\mathbf{B}_{1} & 0 \\
0 & \mathbf{B}_{2} \\
\mathbf{B}_{1} & \mathbf{B}_{2}
\end{array}\right]\left[\begin{array}{c}
\mathbf{U}_{x} \\
\mathbf{U}_{y}
\end{array}\right]
$$

Next, the surface force array $\mathbf{F}_{h k}$ on $\tilde{s}_{h k}$ is

$$
\mathbf{F}_{h k}=\left[\begin{array}{c}
F_{x} \\
F_{y}
\end{array}\right]=\left[\begin{array}{ccc}
\tilde{s}_{x} & 0 & \tilde{s}_{y} \\
0 & \tilde{s}_{y} & \tilde{s}_{x}
\end{array}\right]\left[\begin{array}{c}
\sigma_{x} \\
\sigma_{y} \\
\tau
\end{array}\right]
$$

where $\sigma_{x}, \sigma_{y}$, and $\tau$ are the stress components evaluated in the center of mass of $\tilde{s}_{h k}$.

Now, using Hook's Law between stress and strain components and (17), we obtain

$$
\left[\begin{array}{c}
\sigma_{x} \\
\sigma_{y} \\
\tau
\end{array}\right]=\mathbf{P}\left[\begin{array}{c}
\varepsilon_{x} \\
\varepsilon_{y} \\
\gamma
\end{array}\right]=\mathbf{P}\left[\begin{array}{cc}
\mathbf{B}_{1} & 0 \\
0 & \mathbf{B}_{2} \\
\mathbf{B}_{1} & \mathbf{B}_{2}
\end{array}\right]\left[\begin{array}{c}
\mathbf{U}_{x} \\
\mathbf{U}_{y}
\end{array}\right]
$$

where $\mathbf{P}$, in the case of 2-D plain stress, for isotropic homogeneous material, is given by

$$
\mathbf{P}=\frac{E}{(1+\nu)(1-2 \nu)}\left[\begin{array}{ccc}
1-\nu & \nu & 0 \\
\nu & 1-\nu & 0 \\
0 & 0 & \frac{1-2 \nu}{2}
\end{array}\right]
$$

Next, for element $t$ we may write the local equilibrium equations in the absence of volume forces

$$
\tilde{\mathbf{D}}\left[\begin{array}{ll}
\mathbf{F}_{x} & \mathbf{F}_{y}
\end{array}\right]=0
$$

where $\mathbf{F}_{x}$ is the array of the $F_{x}$ component of the resulting surface force associated with node $n_{i}$, and similarly for $\mathbf{F}_{y}$.

Finally, assembling the contribution from (21) for each $t$, the final system becomes

$$
\mathbf{K}_{M} \mathbf{U}=\mathbf{F}
$$

where $\mathbf{U}=\left[\mathbf{U}_{x}^{T} \mathbf{U}_{y}^{T}\right]^{T}$, and $\mathbf{F}$ is the array of the mechanical loads with nonnull entries on the nodes $n_{i} \in \Sigma_{1}$ only, calculated according to (13); $\mathbf{K}_{M}$ is the resulting stiffness matrix for the elastostatic problem. In addition, the constraints $U_{x}=0, U_{y}=$ 0 are imposed for $n_{i} \in D_{M} \cap \Sigma_{2}$.

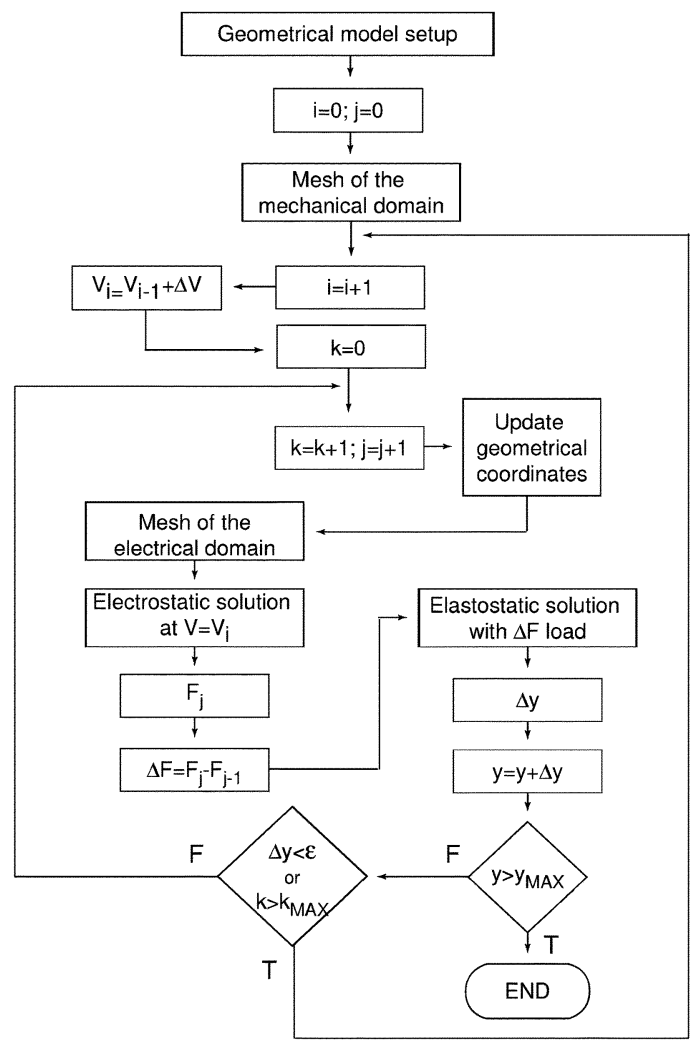

Fig. 3. Flowchart of the sequential field coupling approach implemented with voltage increments. The internal loop (indexed with $k$ ) describes the increments of loads $\Delta F$, while the outer loop (indexed with $i$ ) describes the increments $\Delta V$ applied to the voltage.

\section{Solution Algorithm}

An efficient, fast, and robust relaxation algorithm has been developed to analyze the electromechanical nonlinear problem presented hereafter. The two domains $D_{M}$ and $D_{E}$ mutually influence each other only at the interface, so that an iterative sequential analysis of the two fields can be suitably performed. A sequential field coupling (SFC) approach with voltage increments is adopted. The total potential difference is split into $n$ increments: for each intermediate voltage value $V_{i}$ the coupled analysis is performed by means of the usual SFC method. The equilibrium configuration is achieved for each load step before increasing the applied potential difference, as shown in the scheme reported in Fig. 3.

\section{NUMERICAL RESULTS AND COMPARISONS}

To evaluate the actual effectiveness of the DGA, a comparison has been performed among the nonlinear solutions obtained by the sequential approach with different methods: a special mechanical finite element [8], a commercial FEM code (ANSYS) operating a coupled field analysis and a FEM/BEM approach (FEM for the structure, BEM for the electric domain). It is well known that the physical problem is fully 3-D, mainly because of the distribution of the electrostatic loads on the microbeam. Nevertheless, in this paper, the comparison among different numerical approaches is limited to a plain static nonlinear coupled problem. The aim is to investigate the possibility to build a fast and reliable 2-D model suitable to approximate, with enough accuracy, the actual 3-D behavior of several specimens experimentally tested. A challenging aspect of the proposed approach 


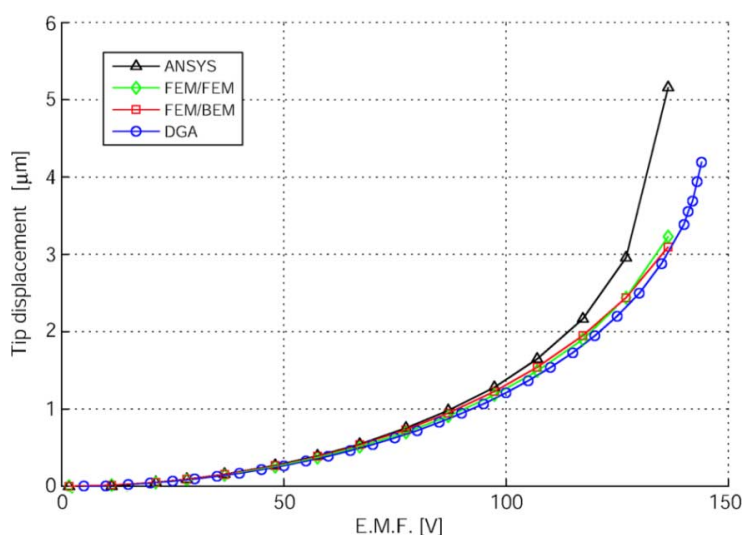

Fig. 4. Numerical comparison of the tip displacement as a function of the applied voltage for the DGA, FEM (ANSYS), sequential nonincremental FEM [8], FEM/BEM approaches $(E=166 \mathrm{GPa}, \nu=0.23, L=200 \mu \mathrm{m}, h=2 \mu \mathrm{m}$, $d=15 \mu \mathrm{m}, g=10 \mu \mathrm{m})$.

is the development of a compact model useful to predict the dynamic behavior of the electromechanical coupled microsystem, where both electromechanical and geometrical nonlinearities are present simultaneously. Several polysilicon cantilever microbeams $(E=166 \mathrm{GPa}, \nu=0.23$ ) have been numerically investigated with length $L \in\{100,200,800 \mu \mathrm{m}\}$, gap $g \in$ $\{10,20,40,100,200 \mu \mathrm{m}\}$, thickness $h=2 \mu \mathrm{m}$, and width $w=15 \mu \mathrm{m}$. The test case has the following nominal (measured) values: $L=200 \mu \mathrm{m}, h=2 \mu \mathrm{m}, w=15 \mu \mathrm{m}, g=10 \mu \mathrm{m}$ ( $L=195 \mu \mathrm{m}, h=2.2 \mu \mathrm{m}, w=15 \mu \mathrm{m}, g=9.4 \mu \mathrm{m}$ ).

In Fig. 4 the tip displacement is presented as a function of the applied voltage up to the pull-in condition. A quite good agreement is shown among the numerical results calculated by DGA (15000 elements and 32000 degrees of freedom for the electrical analysis and 756 elements and 4000 degrees of freedom for the structural analysis), by a FEM (ANSYS) (iterative, mesh morphing, 80 PLANE183 for the solid beam, 3000 PLANE 121 for the electrical analysis), by a sequential nonincremental FEM [8] (3036 elements, 346 nodes along beam boundary for electrical analysis; 41 nodes, 40 Timoshenko two-node beam elements for structural analysis), and by a FEM/BEM (337 twonode boundary elements, 188 nodes along beam boundary for electrical analysis; 31 nodes, 30 two-node Timoshenko beam elements for the structure). The DGA code, developed in Fortran 90 , takes approximately $4 \mathrm{~s}$ to perform the internal loop, indexed with $k$ in Fig. 3, on a 3-GHz Pentium IV, 2-GB RAM; it seems to be promising in the perspective of the implementation into a dynamic solution algorithm including electromechanical and geometrical nonlinearities. A direct comparison of the performance in terms of efficiency and computational time with the other methods is unpractical since they have been developed in different environments (ANSYS, Matlab).

Fig. 5 compares the experimental and numerical predictions from DGA. The difference between the curves obtained with the nominal (solid) and measured (dashed) dimensions is mainly due to the 3-D fringing effects of the electrostatic load distribution. The increment of the force per unit length, computed with a 3-D FEM analysis, is about 1.4. To account for fringing effects by means of a 2-D model, an effective thickness $h_{\text {eff }}$ may be introduced; in our case $h_{\text {eff }}=(2.2 / \sqrt[3]{1.4})=1.97 \mu \mathrm{m}$, which is close to the nominal value.

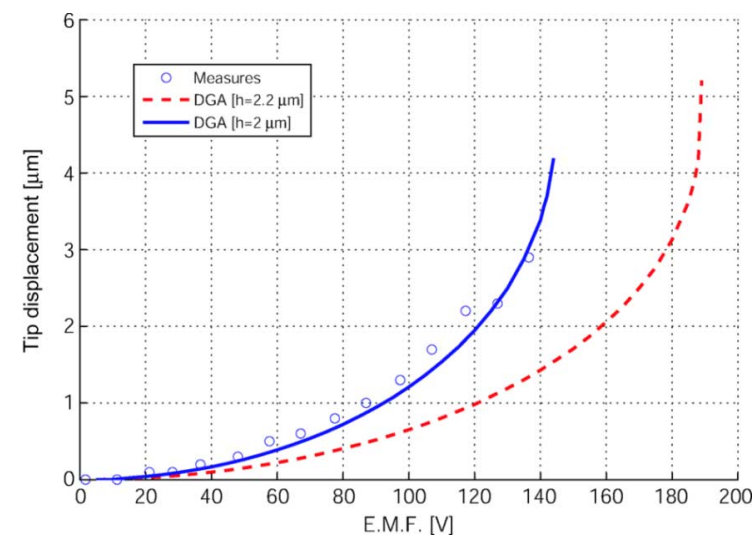

Fig. 5. Comparison of experimental tip displacement with DGA numerical predictions, using measured $(2.2 \mu \mathrm{m})$ and nominal thickness $(2 \mu \mathrm{m})$.

\section{CONCLUSION}

This paper deals with the application of a discrete geometric approach to the numerical prediction of the nonlinear static behavior of electrostatic microactuators. Plain static solution demonstrates that the performance of DGA is competitive and assures the same level of accuracy of available methods like FEM coupled-field solution, sequential nonincremental FEM and FEM-BEM hybrid method. A preliminary experimental validation defined the limits of application of the 2-D modelling, mainly due to the 3-D fringing effect of the electrostatic load distribution. Nevertheless the experimental results are fitted by the 2-D model where an effective microbeam thickness is calibrated on the actual response of the microsystem. This approach could be proposed instead of model reduction techniques to avoid the computational problems related to the solution of 3-D coupled models.

\section{ACKNOWLEDGMENT}

This work was partially founded by the Italian Ministry of University and Scientific Research under Grant 2005/2005091729.

\section{REFERENCES}

[1] F. De Bona and E. Enikov, Microsystems Mechanical Design, ser. CISM Lectures Series. Wien, Germany: Springer-Verlag, 2006.

[2] M. G. Munteanu, F. De Bona, A. Collenz, and E. Brusa, "Geometrical nonlinearities of electrostatically actuated microbeams," in Proc. ECCOMAS, Jyväskylä, Finland, 2004.

[3] E. Tonti, "Algebraic topology and computational electromagnetism," in 4th IMF, 1988, pp. 284-294.

[4] A. Bossavit, "How weak is the weak solution in finite elements methods?," IEEE Trans. Magn., vol. 34, pp. 2429-2432, 1998

[5] E. Tonti, "Finite formulation of electromagnetic field," IEEE Trans. Magn., vol. 38, pp. 333-336, 2002.

[6] P. Bettini and F. Trevisan, "Electrostatic analysis for plane problems with finite formulation," IEEE Trans. Magn., vol. 39, pp. 1127-1130, 2003.

[7] A. Somà, A. Collenz, F. De Bona, and A. Gugliotta, "Large deflections of microbeams under electrostatic loads," J. Micromech. Microeng., vol. 14, pp. 365-373, 2004.

[8] E. Brusa and M. Munteanu, "Coupled-field FEM nonlinear dynamics analysis of continuous microsystems by non incremental approach," Analog Integr. Circuits Signal Process., vol. 48, pp. 7-14, 2006.

[9] A. Bossavit and L. Kettunen, "Yee-like schemes on staggered cellular grids: A synthesis between FIT and FEM approaches," IEEE Trans. Magn., vol. 36, no. 4, pp. 861-867, 2000.

Manuscript received June 24, 2007. Corresponding author: P. Bettini (e-mail: bettini@uniud.it). 